\title{
Deep brain stimulation for the treatment of Parkinson's disease: subthalamic nucleus versus globus pallidus internus
}

\author{
M Krause, W Fogel, A Heck, W Hacke, M Bonsanto, C Trenkwalder, V Tronnier
}

\begin{abstract}
Objectives-Deep brain stimulation of the basal ganglia has become a promising treatment option for patients with Parkinson's disease who have side effects from drugs. Which is the best target-globus pallidus internus (GPi) or subthalamic nucleus (STN)-is still a matter of discussion. The aim of this prospective study is to compare the long term effects of GPi and STN stimulation in patients with severe Parkinson's disease.

Patients and methods-Bilateral deep brain stimulators were implanted in the GPi in six patients and in the STN in 12 patients with severe Parkinson's disease. Presurgery and 3, 6, and 12 months postsurgery patients were scored according to the CAPIT protocol.

Results-Stimulation of the STN increased best Schwab and England scale score significantly from 62 before surgery to 81 at 12 months after surgery; GPi stimulation did not have an effect on the Schwab and England scale. Stimulation of the GPi reduced dyskinesias directly whereas STN stimulation seemed to reduce dyskinesias by a reduction of medication. Whereas STN stimulation increased the unified Parkinson's disease rating scale (UPDRS) motor score, GPi stimulation did not have a significant effect. Fluctuations were reduced only by STN stimulation and STN stimulation suppressed tremor very effectively.

Conclusion-Stimulation of the GPi reduces medication side effects, which leads to a better drug tolerance. There was no direct improvement of bradykinesia or tremor by GPi stimulation. Stimulation of the STN ameliorated all parkinsonian symptoms. Daily drug intake was reduced by STN stimulation. The STN is the target of choice for treating patients with severe Parkinson's disease who have side effects from drugs.
\end{abstract}

(F Neurol Neurosurg Psychiatry 2001;70:464-470)

Keywords: Parkinson's disease; deep brain stimulation; globus pallidus internus; subthalamic nucleus

Twenty five years after the introduction of effective pharmacotherapy with levodopa, neurosurgical approaches are experiencing a revival in the treatment of Parkinson's disease. Two reasons account for this change in therapeutic strategy. On the one hand, many patients with Parkinson's disease who have been treated with levodopa and dopamine agonists for many years do not respond adequately to pharmacological therapy any more and also show side effects of the drug, such as motor fluctuations or dyskinesias. On the other hand, thanks to the new technique of high frequency stimulation, neurosurgical therapy has become much safer, especially with bilateral implantation. In accordance with experience gained with lesioning, at first the stimulating leads were placed in the globus pallidus internus (GPi.). ${ }^{1-3}$ Although lesions in the subthalamic nucleus (STN) in monkeys treated with 1-methyl-4-phenyl-1,2,3,6-tetrahydropyridine (MPTP) yielded a much greater improvement in all symptoms of Parkinson's disease, ${ }^{4-8}$ this method was associated with severe side effects such as hyperkinetic, ballistic movement disorders. Because the extent of side effects can be controlled by adjusting the stimulator variables, the STN was chosen as a new target for stimulation to abolish akinesia and rigidity. ${ }^{9-11}$ Which target for stimulation is best, the GPi or the STN, has yet to be determined. The aim of this prospective, non-randomised study was to compare the long term efficacy and safety of GPi and STN stimulation in patients with severe Parkinson's disease.

\section{Patients and methods}

Since 1995 bilateral deep brain leads have been implanted in 33 patients in either the GPi or in the STN. A prospective non-randomised trial was performed to compare both stimulation targets. The first six patients were assigned to the GPi group, followed by six further patients assigned to the STN group. These patients were matched for age and disease duration. After an intermediate evaluation of these 12 patients, GPi stimulation was abandoned and all further patients were implanted in the STN. This paper presents the long term results of the first 18 patients with a follow up of at least 1 year.

Inclusion criteria were established according to the CAPIT protocol. ${ }^{12}$ All patients had advanced Parkinson's disease (off state Hoehn and Yahr >2.5). Patient characteristics are given in table 1 .

Table 1 Patient details

\begin{tabular}{lcll}
\hline $\begin{array}{l}\text { Stimulation } \\
\text { target }\end{array}$ & $\begin{array}{l}\text { No of } \\
\text { patients }\end{array}$ & $\begin{array}{l}\text { Mean age }(y) \\
\text { (min/max) }\end{array}$ & $\begin{array}{l}\text { Mean disease } \\
\text { duration }(y) \\
\text { (min/max) }\end{array}$ \\
\hline GPi & $6^{\star}$ & $58.5(46 / 65)$ & $14.7(5 / 20)$ \\
STN & $12^{\star}$ & $58.7(45 / 69)$ & $13.7(8 / 23)$ \\
\hline
\end{tabular}

Gpi=Globus pallidus internus; STN=subthalamic nucleus. Age and disease duration are at time of surgery.

${ }^{\star}$ In each group one patient was lost to follow up. 
DESIGN

One week before surgery and 3, 6, and 12 months after surgery ( \pm 2 weeks) patients were examined and scored according to the CAPIT protocol $^{12}$ by an experienced neurologist. All motor scores were videotaped and evaluated later by a neurologist blinded to the study. Mean values of both neurologists was taken.

Scores from the unified Parkinson's disease rating scale (UPDRS) ${ }^{13}$ and the Obeso dyskinesia scale were ascertained, as were age of the patient, disease onset, and medication. Presurgery part III (motor score) of the UPDRS and the Obeso dyskinesia intensity scale ${ }^{12}$ were applied under practically defined drug on and drug off conditions. ${ }^{12}$ After surgery, these scores were ascertained in four conditions: medication off and stimulator off; drug off and stimulator on; drug on and stimulator off; and drug on and stimulator on.

The Hoehn and Yahr ${ }^{14}$ and the Schwab and England scale ${ }^{15}$ were then applied and the best and worst of each time of testing recorded, part I, mentation, part II, activities of daily living (ADL), and part IV, complications, fluctuations, and dyskinesia, of the UPDRS were taken as a single anamnestic value for the previous week.

\section{OPERATION}

A stereotatic technique was employed for implantation, using the Zamorano-Dujovny stereotactic system (Stryker-Leibinger, Freiburg, Germany), guided by MRI. The surgical procedure was performed after the patient received a local anaesthetic in standby anaesthesia with intravenous, conscious sedation and analgesia during application of the frame. No further medication was given during the imaging, microrecording, and stimulation phases of the operation. An MRI compatible frame was fitted to the head according to external landmarks, so as to be as parallel as possible to the intercommissural (IC) line. Magnetic resonance imaging was performed using a 1.5 Tesla scanner (Edge, Picker International, USA). A midsagittal image was acquired and the deviation of the frame to the IC line was determined. Axial slices were then obtained parallel to the IC plane. The pallidal target was chosen $2-3$ $\mathrm{mm}$ anterior to the midcommissural point, 19-22 mm lateral the midline and 3-6 $\mathrm{mm}$ below the IC line. The target was corrected using the angle deviation between the frame and IC line.

The subthalamic target was chosen 10-12 $\mathrm{mm}$ lateral and $2-3 \mathrm{~mm}$ posterior to the midcommissural point and $2-4 \mathrm{~mm}$ below the AC-PC line.

Two precoronal burr holes were drilled 2.5 $\mathrm{cm}$ lateral of the midline. This created a trajectory between 3 and 10 degrees with reference to the sagittal plane for $\mathrm{GPi}$, between 8 and 16 degrees with reference to the sagittal plane for STN, and between 45 and 60 degrees with reference to the AC-PC line for both targets.

Concentric bipolar tungsten microelectrodes (impedance $0.5-1 \mathrm{MOhm}$, tip 1-2 $\mu \mathrm{m}$ diameter, Inomed, Teningen, Germany) and a hydraulic microdrive (Trent-Wells, Coulter- ville, USA) were used to take the recordings, beginning $10 \mathrm{~mm}$ above the target. Single unit activity was recorded using conventional amplifiers (Toennies, Fa Jaeger, Germany) with a bandwidth of $30-10000 \mathrm{~Hz}$ and amplified 10000 times. Analog data were displayed on an oscilloscope (Digital storage oscilloscope VC 6275, Hitachi Denshi, Rodgau, Germany) and stored on magnetic tape. Further data were simultaneously recorded via an A/D amplifier (Brain Wave Systems Corporation) and digitalised for later analysis with analysis software (Data Wave Technologies Corp, Longmont, CO, USA).

\section{$G P i$}

After determining the boundary of the internal segment of the GPi by enhanced frequency of the spontaneous neuronal activity, single unit discharges were tested for their response to passive or active movements. After changes in background noise and cessation of rapid discharges, microstimulation and macrostimulation was carried out to delineate the optic tract. When an optic response was elicited with macrostimulation - for example, white stars or coloured bands (phosphenes), usually in the lateral visual field between 0.5 and $1 \mathrm{~V}$ showing close proximity to the optic tract, the electrode was corrected to optimise the intraoperative neurophysiological responses. For intraoperative macrostimulation and chronic stimulation a quadropolar electrode (Mod 3387 DBS, Medtronic Inc, Minneapolis, USA) was used. A combination of four electrodes with a length of $1.5 \mathrm{~mm}$, a diameter of $1.2 \mathrm{~mm}$, and an interelectrode distance of $1.5 \mathrm{~mm}$ between electrodes made it possible to stimulate the structure within a distance of $10.6 \mathrm{~mm}$ using different monopolar or bipolar stimulation modes to determine the optimal target for stimulation.

\section{$S T N$}

Approaching the STN, the trajectory first crossed the ventraloralis anterior or recticular thalamic nucleus, disclosing typical thalamic activity with high amplitude, low frequency singular or bursting cells. By lowering the electrode towards the zona incerta only fibre activity was recorded and when entering the STN, typically, neurons were found to be firing with high frequency compared with thalamic activity $(30-60 \mathrm{~Hz})$, sometimes with bursting features according to tremor frequency. The boundaries of the STN are the internal capsule laterally and anteriorly and the $\mathrm{H}_{2}$ field of Forel medially.

Macrostimulation showed reduction in regidity and improvement in bradykinesia. In a few cases hyperkinesias were accomplished contralateral to the stimulation site. Side effects by stimulation were dystonic movements or paraesthesias, if the electrodes were placed too laterally, eye bulb deviation if placed too medially, and paraesthesias if placed too posteriorly (lemniscal fibres). For STN stimulation a lead with reduced electrode interspace of $0.5 \mathrm{~mm}$ was implanted (Mod 3389 DBS, Medtronic Inc, Minneapolis, USA). 
Table 2 Mean (SEM) UPDRS subscores

\begin{tabular}{|c|c|c|c|c|c|}
\hline & \multirow[b]{2}{*}{$\begin{array}{l}\text { Stimulus } \\
\text { target }\end{array}$} & \multicolumn{4}{|c|}{ UPDRS subscore } \\
\hline & & Preoperative & $\begin{array}{l}3 \text { months } \\
\text { postoperative }\end{array}$ & $\begin{array}{l}6 \text { months } \\
\text { postoperative }\end{array}$ & $\begin{array}{l}12 \text { months } \\
\text { postoperative }\end{array}$ \\
\hline \multirow[t]{2}{*}{ Mentation } & GPi & $5.2(1.0)$ & $2.6(0.5)$ & $3.2(0.9)$ & $2.6(0.7)$ \\
\hline & STN & $3.3(0.7)$ & $3.4(0.9)$ & $2.5(0.9)$ & $4.1(1.1)$ \\
\hline \multirow[t]{2}{*}{$\mathrm{ADL}$} & GPi & \multirow{2}{*}{$\begin{array}{l}17.4(1.5) \\
23.7(3.3)\end{array}$} & \multirow{2}{*}{$\begin{array}{l}13.8(1.3) \\
14.6(2.8)\end{array}$} & \multirow{2}{*}{$\begin{array}{l}18.0(1.4) \\
19.2(3.1)\end{array}$} & \multirow{2}{*}{$\begin{array}{l}16.6(2.4) \\
17.5(2.8)\end{array}$} \\
\hline & STN & & & & \\
\hline \multirow[t]{2}{*}{ Complications } & GPi & \multirow{2}{*}{$\begin{array}{l}2.0(0.5) \\
1.5(0.3)\end{array}$} & $1.2(0.6)$ & $1.2(0.4)$ & $2.0(0.3)$ \\
\hline & STN & & $1.4(0.3)$ & $1.2(0.2)$ & $1.3(0.3)$ \\
\hline \multirow{2}{*}{$\mathrm{H}$ and $\mathrm{Y}$ worst } & GPi & $3.8(0.6)$ & $3.2(0.4)$ & $3.8(0.5)$ & $4.3(0.5)$ \\
\hline & STN & $4.4(0.2)$ & $4.0(0.2)$ & $4.2(0.2)$ & $4.3(0.3)$ \\
\hline \multirow[t]{2}{*}{$\mathrm{H}$ and $\mathrm{Y}$ best } & GPi & \multirow{2}{*}{$\begin{array}{l}2.7(0.3) \\
3.2(0.3)\end{array}$} & \multirow{2}{*}{$\begin{array}{l}2.7(0.4) \\
2.2(0.3)\end{array}$} & \multirow{2}{*}{$\begin{array}{l}2.7(0.2) \\
2.5(0.3)\end{array}$} & \\
\hline & STN & & & & $2.5(0.2)$ \\
\hline$S$ and $E$ worst & GPi & $42 \quad(6.6)$ & $54 \quad(6.0)$ & $38 \quad(9.7)$ & $48 \quad(8.0)$ \\
\hline & STN & $34 \quad(6.9)$ & $34 \quad(7.1)$ & $41 \quad(7.4)$ & $36 \quad(7.5)$ \\
\hline$S$ and $E$ best & GPi & $76 \quad(6.8)$ & $80 \quad(3.2)$ & $72 \quad(4.9)$ & $78 \quad(5.8)$ \\
\hline & STN & $62 \quad(6.7)$ & $83 \quad(5.2)$ & $77 \quad(6.9)$ & $81 \quad(4.6)$ \\
\hline
\end{tabular}

GPi=Globus pallidus internus; $\mathrm{STN}=$ subthalamic nucleus; $\mathrm{H}$ and $\mathrm{Y}=$ Hoehn and $\mathrm{Yahr}$; $\mathrm{S}$ and $\mathrm{E}=\mathrm{Schwab}$ and England.

In most patients one track per side was sufficient to reach the target. Some patients needed two tracks; one patient needed three tracks on one side to achieve a good perioperative reduction of rigidity and alleviation of bradykinesia. The final decision for electrode placement was mainly based on the perioperative reduction of rigidity, which turned out to be the most stable symptom. Thresholds were determined and the position with the lowest threshold needed to abolish rigidity without producing side effects was used as the final electrode position. Postoperative MRI was performed in all patients. Due to the large electrode artifact (in the range of 3-4 $\mathrm{mm}$ ) only landmarks such as the red nucleus (for the STN) or the optical tract and the medial border of the GPi (for the GPi) were used to estimate the accuracy of the electrode placement. Therefore, coordinates provided in the paper were the stereotactic coordinates used during surgery.

After temporary extension of the leads and connection to two external screeners, a test trial of 7-10 days was conducted under

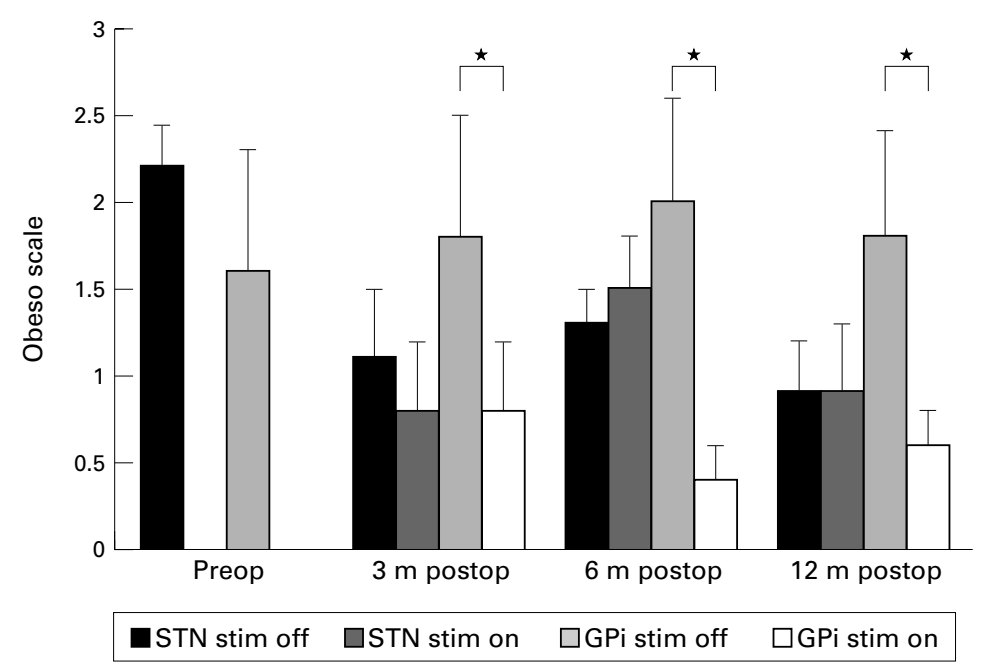

Figure 1 Obeso dyskinesias. Preop=presurgery; $x m$ posto $p=x$ months after stimulator implant; stim off=stimulator off; stim on=stimulator on; $S T N=$ subthalamic nucleus; GPi $=$ globus pallidus internus; ${ }^{\star} p<0.05$. antibiotic treatment. If results were satisfactory in the test period two stimulators were implanted.

\section{STATISTICS}

For all data the median and mean and standard error (SEM) were calculated and the Wilcoxon rank sum test for paired data was applied.

A difference was considered significant when error probability was less than 0.05 .

\section{Results}

The number and age of patients and duration of disease are given in table 1 . In each group there was one patient lost to follow up (see complications).

Mean values (SEM) of the UPDRS subscore mentation (UPDRS item 1-4), activities of daily living (UPDRS item 5-17), and complications (UPDRS item 40-42), as well as Hoehn and Yahr and the Schwab and England scales are presented in table 2 .

Although there was a mean improvement in all scores during most follow up examinations, only the 3 and 12 month follow up data of the Schwab and England best of the STN group reached significance.

Fluctuations were reduced significantly by STN stimulation from 4.2 (SEM 0.3) before surgery to 1.5 (SEM 0.3) 6 and 12 months after surgery according to the UPDRS (items 36-39). On the other hand GPi stimulation did not reduce fluctuations at all.

Dyskinesias were measured with the single value UPDRS subscore (items 32-35) and with the Obeso dyskinesia intensity scale. By contrast with the UPDRS subscore, which is an anamnestic score, the Obeso scale (fig 1) was ranked in the medication and stimulator off and on state. In the UPDRS subscore there was a nearly equivalent reduction of dyskinesias in both groups. GPi stimulation reduced dyskinesias from 6.2 (SEM 3) before surgery to 1.8 (SE 0.8) 3 months and 2.6 (SEM 1.4) 12 months after surgery. As well as reduced STN stimulation the UPDRS dyskinesia subscore was reduced from 7.3 (SEM 1.0) before surgery to 1.8 (SEM 0.6) 3 months and 3.1 (SEM 1.1) 12 months after surgery.

The Obeso score (fig 1), however, showed a direct effect of the stimulation in the GPi group while in the medication on state. Stimulation in the STN group did not reduce dyskinesias directly as the GPi stimulation did. Stimulation of the STN seemed to reduce dyskinesias secondarily by reducing drug intake (see medication).

In two patients ballistic hyperkinesias occurred in the STN group depending on stimulation intensity. Both patients took more than $1000 \mathrm{mg}$ levodopa and had severe drug induced dyskinesias before surgery. Directly after surgery, stimulation with more than $1 \mathrm{~V}$ induced ballistic movement disorders in these patients. This side effect ceased after several weeks of chronic stimulation and they were able to reduce their daily levodopa intake dramatically. Yet there was not a clear correlation between drug induced hyperkinesias presurgery and ballistic movement disorders postsurgery. 


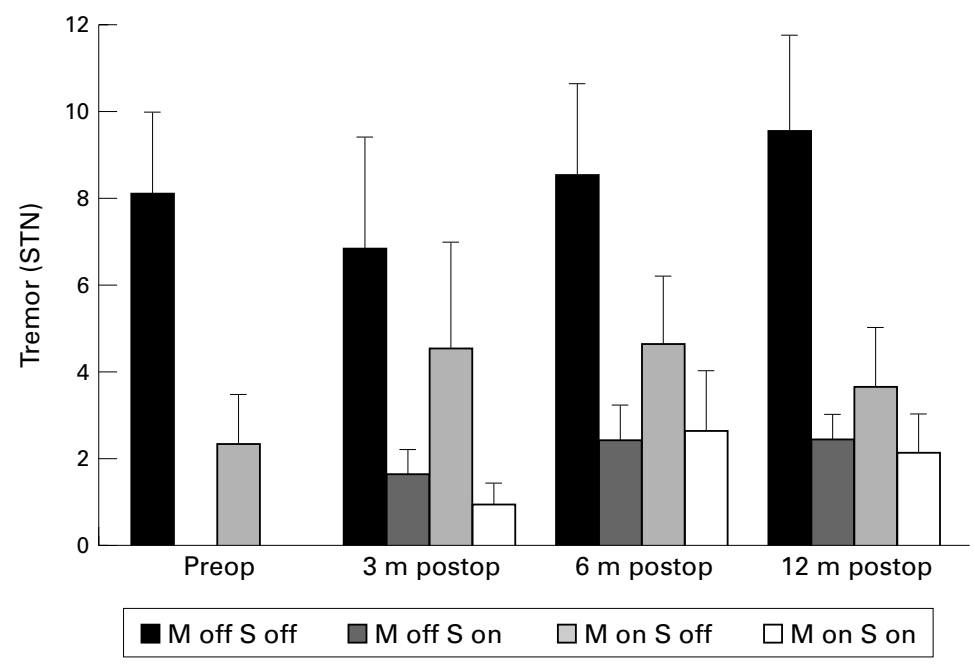

Figure 2 Tremor in STN group. Preop=presurgery; $x m$ postop $=x$ months after stimulator implant; $S$ off=stimulator off; $S$ on=stimulator on; $M$ off= without medication; $M$ on=after levodopa intake.

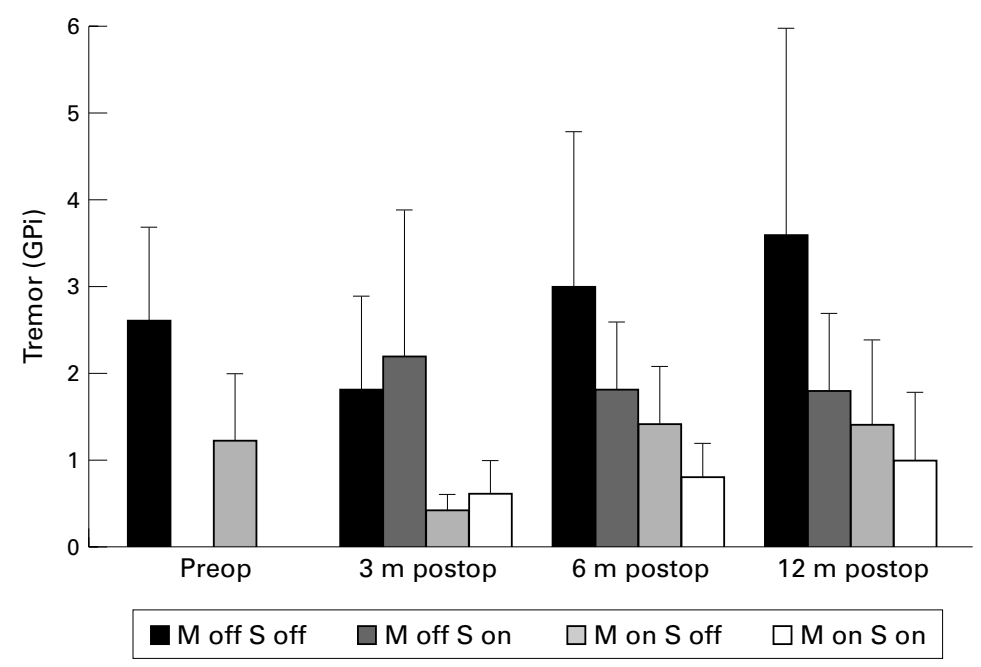

Figure 3 Tremor in GPi group. For explanation see fig 2.

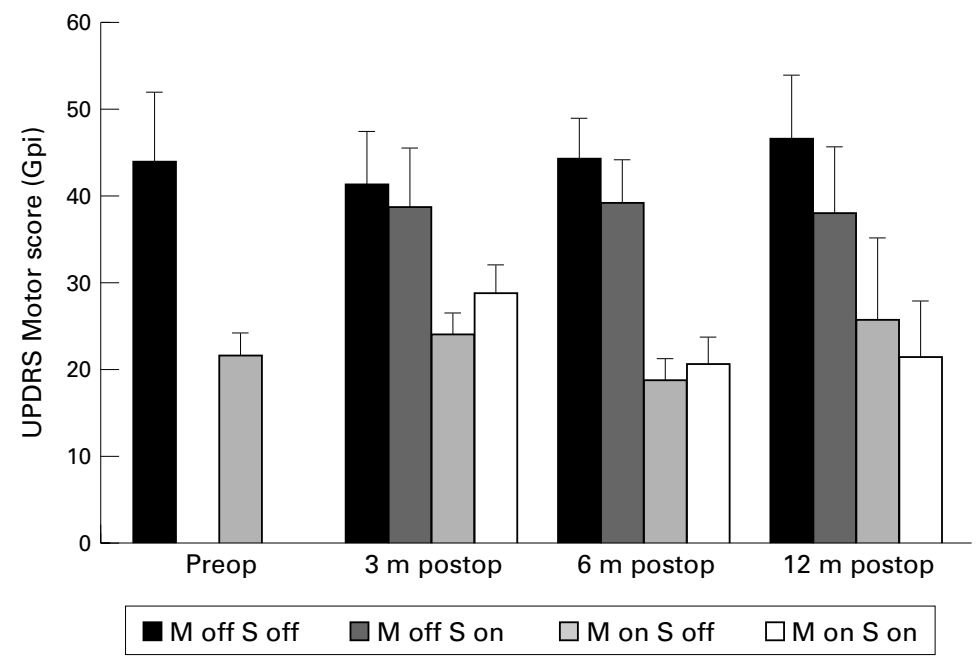

Figure 4 UPDRS motor score in GPi group. For explanation see fig 2

In figures 2 and 3 all tremor related items of the UPDRS (items 20 and 21) are presented. Stimulation of the STN suppressed tremor much better than medication (fig 2) whereas stimulation of the GPi did not have a significant effect on tremor (fig 3). There was a strong correlation between stimulation intensity and tremor suppression in the STN group.

The UPDRS motor subscore (items 18-31) is shown in figures 4 and 5 . Turning on the stimulator in the STN group improved the UPDRS motor score significantly in the medication off state. Improvement in the medication on state did not reach significance (fig 4 ), and neither did mean improvement of the motor score in the GPi group (fig 5).

Because medication was changed quite often, we calculated an equivalent dose for dopamine agonists. Based on various recommendations of the pharmaceutical industry the defined equivalent values referring to $100 \mathrm{mg}$ levodopa were $1 \mathrm{mg}$ apomorphine and $10 \mathrm{mg}$ bromocriptine, $1.5 \mathrm{mg}$ carbergoline, $20 \mathrm{mg}$ dihydroergocriptine, $0.7 \mathrm{mg}$ lisurid, $1 \mathrm{mg}$ pergolid and pramipexol, and $4 \mathrm{mg}$ ropinirol. The slow release form of levodopa was added at a two thirds rate. COMT inhibitors were presented separately.

Although in the GPi group levodopa intake increased significantly over the course of 1 year after stimulator implant from an average of 277 mg (SEM 123) to $772 \mathrm{mg}$ (SEM 138), the levodopa intake in the STN group was stable at an average of about $400 \mathrm{mg}$ a day (SEM 182). In the STN group the dopamine agonists were reduced significantly by one third; from 542 $\mathrm{mg}$ (SEM 154) levodopa equivalent to $360 \mathrm{mg}$ (SEM 112) and COMT inhibitors were reduced from $100 \mathrm{mg}$ (SEM 68) to $30 \mathrm{mg}$ (SEM 30). In the GPi group the dopamine agonists intake was stable at an average of 205 mg (SEM 123) levodopa equivalent dose a day and COMT inhibitors were even increased by an average of $75 \mathrm{mg}$ (SEM 54) a day.

\section{COMPLICATIONS}

Acute complications developed in one patient (STN stimulation). While introducing the right lead a basal ganglia and ventricular haemorrhage occurred, which led to an acute, mild hemiparesis on the left side. The intraventricular haemorrhage had to be treated with a temporary external ventriculostomy. The patient recovered within 2 months and was discharged without any significant impairment. The lead had dislocated and so the patient was excluded from further testing.

In the GPi group one woman dropped out because of a severe depression for which she had to remain in hospital for more than a year. She refused further participation in the study. Data from this patient were excluded from all statistical procedures.

Three patients (two with GPi and one with STN stimulation) reported a strong increase in libido. Two of these patients had to be treated in an inpatient psychiatric clinic for this reason.

Many patients reported transient dysaesthesias when turning on the stimulator. Patients with a GPi stimulator reported visual irritation at certain intensity levels. None of the patients experienced a reduction in their visual field (according to Goldmann perimetry). Two patients with GPi stimulation developed severe dysarthria; one of them was not able to speak 


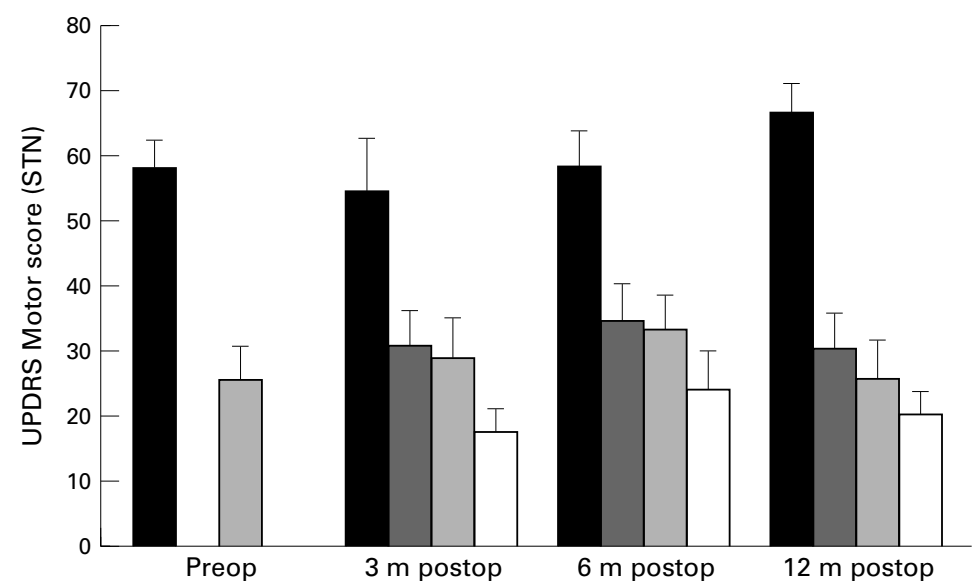

M off S off $\square$ M off S on $\square$ M on S off $\square$ M on S on

Figure 5 UPDRS motor score in STN group. For explanation see fig 2.

understandably any more. This side effect was not dependent on stimulation. Two patients in the STN group also reported stimulation related dysarthria, which, however, was very moderate.

One patient (GPi group) developed a severe psychosis more than 1 year after stimulator implant. This episode was closely related to the introduction of tolcapone in his therapy.

\section{Discussion}

With the introduction of high frequency stimulation instead of lesioning in 1987 by Benabid et $a l^{16}{ }^{17}$ the surgical treatment of Parkinson's disease became much safer and new targets were chosen. High frequency stimulation is gaining in popularity, yet only very few study groups have investigated the long term results of these stimulators and prospectively evaluated results of stimulation in different targets are scarce.

Bergman et al demonstrated in 1990 that in monkeys treated with MPTP STN lesioning resulted in an outstanding improvement of all parkinsonian symptoms, but was associated with severe side effects. ${ }^{5}$ Pallidal lesions and stimulation in humans yielded very divergent results. One study even reported a proakinetic effect when stimulating more caudally within the Gpi. ${ }^{18}{ }^{19}$ Others reported a good improvement in motor function. ${ }^{20} 21$

According to our results chronic STN stimulation came out superior to GPi stimulation for most of the scores we examined. Only dyskinesias were reduced to a greater extent by GPi stimulation; this effect occurred directly when the GPi stimulator was turned on. However, by reducing dyskinesias, bradykinesia was induced. Although some patients in the GPi group were able to walk with hyperkinesias, turning the GPi stimulator on resulted in a decrease of hyperkinesias but severe freezing prevented walking and markedly decreased motor function. When turning the GPi stimulator off, dyskinesias appeared with a latency of minutes to hours. This implies a delayed effect of high frequency stimulation, probably due to reorganisation of basal ganglia loops involved in the pathogenesis of dyskinesias.
Stimulating the STN also had a direct effect on hyperkinesias, inducing delayed (minutes up to 2 hours) hyperkinetic, ballistic movements in most patients of the STN group. This effect occurred mainly contralateral to the stimulation site and was more pronounced with higher stimulation intensity. This occurrence of hyperkinesias with high stimulation intensities fits well into the current basal ganglia loop concept ${ }^{22}$ with inhibition of the STN causing underactivity of the GPi nucleus. This underactivity results in reduced thalamic inhibition with consecutive hyperactivity of prefrontal cortical areas. Interestingly, the prodyskinetic effect habituates with longer lasting stimulation and the stimulation intensity can be slowly (within days to weeks) increased without causing further hyperkinesias. One explanation could be an increasingly reduced sensitivity of the neurons within the GPi receiving projections from the STN. Alternatively there might be plasticity within the basal ganglia loops inhibiting overshooting output to cortical neurons. As a final result hyperkinesias were also reduced within the STN group (fig 1). Presumably this is due to the reduced dopaminergic medication made possible by the antiakinetic effect of STN stimulation.

These results show how differently STN and GPi stimulation work. Although GPi stimulation mainly reduces side effects of antiparkinsonian drugs, creating a higher drug tolerance, STN stimulation ameliorates the actual parkinsonian symptoms, leading to lower drug requirements. By contrast with other studies, ${ }^{20}{ }^{21}$ we did not see a relief in parkinsonian off symptoms as a result of GPi stimulation, particularly not in hypokinesia or tremor. This difference in results might be attributed to different targets within the GPi. A rather more dorsal and lateral stimulation in the GPi in comparison with our target choice might produce a better alleviation of parkinsonian off symptoms. ${ }^{19}$

The antiparkinsonian effect of STN stimulation was comparable with levodopa (fig 5). One patient in the STN group could even stop taking levodopa and dopamine agonists for several weeks. Stimulation of the STN mainly improves off drug symptoms, whereas the improvement of the drug on state was moderate. There is a marked reduction of fluctuations with a decrease of duration and severity of the off states.

Stimulation of the STN suppresses tremor as effectively as stimulation of the ventral intermediate part of the thalamic nucleus (VIM). ${ }^{23}{ }^{24}$ In opposition to chronic stimulation in the VIM, switching off the stimulator did not cause a tremor rebound and no change in tremor morphology was seen. ${ }^{23}$ Therefore, STN stimulation might be the preferred target even for tremor dominant Parkinson's disease, especially as chronic VIM stimulation is only effective for tremor reduction but does not ameliorate other parkinsonian symptoms.

A main problem of early studies was the comparability of the results. ${ }^{25-30}$ Improvement was poorly documented and always highly subjective. A crucial point in evaluating the effect 
of surgery in Parkinson's disease is the study design and the testing itself. A step forward towards unbiased evaluation was the development of the CAPIT protocol in $1992^{12}$, which was designed to compare studies investigating transplantation surgery in Parkinson's disease. However, testing according to this protocol is very time consuming and requires a high degree of compliance from the patient and the investigator.

This study complied with the CAPIT protocol, with some exceptions. UPDRS part I (mentation), and part II (ADL) scores were not differentiated in medication on and off conditions but were recorded as an average score evaluated retrospectively for the week before UPDRS testing. It seems to us that an average amnestic ADL and mentation value would more accurately reflect the effect of the stimulation as it reflects functional changes in activities of daily living more accurately than the separation in drug on and off states.

One problem of this protocol, which was already discussed by Langston et al in $1992^{12}$, is the definition of drug off and on states. The participants of the CAPIT consensus conference tried to establish the practical defined off state as a 12 hour drug withdrawal. Twelve hours were probably practical at that time; however, with the introduction of new longer lasting dopamine agonists such as cabergolin or pramipexol this definition has become at least questionable. Therefore, long lasting dopamine agonists such as cabergoline were withdrawn for at least 48 hours in this study.

A further problem is evaluating the effect of stimulation on drug intake, as our patients changed their medication rather often. This change did not necessarily reflect a disease activity but was often closely related to the introduction of new drugs such as the COMT inhibitors. To our knowledge only very few studies have investigated equivalent doses of dopamine agonists. We calculated equivalent doses, being well aware that this procedure will be subject to criticism. Even so, these equivalent doses should give an overall impression of whether stimulation reduces or increases drug intake.

Perioperative complications occurred in only one patient, which is in agreement with the literature. Minor side effects, such as transient dysaesthesias, occurred in both groups when turning on the stimulator or programming the stimulator. The hyperkinetic, ballistic movements, which occurred in some patients with STN stimulation was related to stimulation intensity. This side effect ceased totally within weeks or months of chronic stimulation.

Side effects as a result of long term stimulation occurred more often in the GPi group and were more severe than in the STN group. Dysarthria in particular, was very debilitating for some patients.

Hypersexuality is known as a side effect of dopaminergic therapy in Parkinson's disease. ${ }^{31}$ To our knowledge an increase of libido has not been reported as a side effect of deep brain stimulation. Whether it is a true increase of libido or rather a change in mood and person- ality due to the influence of the STN and GPi on the limbic system is unclear. Further studies are warranted to investigate this phenomenon.

\section{Conclusion}

High frequency stimulation of the STN and the GPi is safe. The effects are stable even after 1 year of stimulation.

Stimulation of the GPi reduces medication side effects, which leads to better drug tolerance. We did not find a direct effect in improving akinesia or tremor.

Stimulation of the STN directly ameliorates all parkinsonian symptoms, and effectively suppresses tremor, without a tremor rebound.

In our opinion, STN stimulation is the target of choice to treat patients with fluctuating Parkinson's disease or medication induced dyskinesias once medication fails. By reducing total drug intake, STN stimulation reduces drug side effects and might even be interesting from an economic point of view. However, the number of patients is small in our study and unequal between the GPi and STN groups and, therefore, larger series should be studied.

1 Siegfried J, Lippitz B. Bilateral chronic electrostimulation of ventroposterolateral pallidum: a new therapeutic approach for alleviating all parkinsonian symptoms. Neurosurgery 1994;35:1126-9.

2 Iacono RP, Lonser RR, Maeda G, et al. Chronic anterior pallidal stimulation for Parkinson's disease. Acta Neurochir Wien 1995;137:106-12.

3 Krack P, Pollak P, Limousin P, et al. Subthalamic nucleus or internal pallidal stimulation in young onset Parkinson's disease. Brain 1998;121:451-7.

4 Bergman $\mathrm{H}$, Wichmann T, DeLong MR. Reversal of experimental parkinsonism by lesions of the subthalamic nucleus. Science 1990;249:1436-8.

5 Aziz TZ, Peggs D, Sambrook MA, et al. Lesion of the subthalamic nucleus for the alleviation of 1-methyl-4phenyl-1,2,3,6-tetrahydropyridine (MPTP)- induced parkinsonism in the primate. Mov Disord 1991;6:288-92.

6 Guridi J, Luquin MR, Herrero MT, et al. The subthalamic nucleus: a possible target for stereotaxic surgery in Parkinson's disease. Mov Disord 1993;8:421-9.

7 Guridi J, Herrero MT, Luquin R, et al. Subthalamotomy improves MPTP-induced parkinsonism in monkeys. Stereotact Funct Neurosurg 1994;62:98-102.

8 Aziz TZ, Peggs D, Agarwal E, et al. Subthalamic nucleotomy Aziz TZ, Peggs D, Agarwal E, et al. Subthalamic nucleotomy
alleviates parkinsonism in the 1-methyl-4-phenyl-1,2,3,6alleviates parkinsonism in the 1 -methyl-4-phenyl- $1,2,3,6-$
tetrahydropyridine (MPTP)- exposed primate. Br f Neurosurg 1992;6:575-82.

9 Burg 1992;6:575-82. effects of subthalamic nucleus stimulation in Parkinson's disease. Stereotact Funct Neurosurg 1994;62:76-84.

10 Limousin P, Pollak P, Benazzouz A, et al. Effect of parkinsonian signs and symptoms of bilateral subthalamic nucleus stimulation. Lancet 1995;345:91-5.

11 Limousin P, Pollak P, Benazzouz A, et al. Bilateral subthalamic nucleus stimulation for severe Parkinson's disease. Mov Disord 1995;10:672-4.

12 Langston JW, Widner H, Goetz CG, et al. Core assessment program for intracerebral transplantations (CAPIT). Mov Disord 1992;7:2-13.

13 Fahn S, Elton R, Committe aMotUD. Unified Parkinson's disease rating scale. In: Fahn S, Marsden C, Goldstein M, et al, eds. Recent developments in Parkinson's disease. 1st ed. New York: Macmillan, 1987:153-63.

14 Hoehn MM, Yhar MD. Parkinsonism: onset, progression, and mortality. Neurology 1967;17:427-42.

15 Schwab RS, England AC. Projection technique for evaluating surgery in Parkinson's disease. Third symposium on Parkinson's disease. Edinburgh: Gillingham and Donaldson, 1969.

16 Benabid AL, Pollak P, Louveau A, et al. Combined (thalamotomy and stimulation) stereotactic surgery of the VIM thalamic nucleus for bilateral Parkinson disease. Appl Neurophysiol 1987;50:344-6.

17 Benabid AL, Pollak P, Hommel M, et al. Treatment of Parkinson tremor by chronic stimulation of the ventral intermediate nucleus of the thalamus. Rev Neurol Paris 1989;145:320-3.

18 Krack P, Pollak P, Limousin P, et al. Levodopa-inhibiting effect of pallidal surgery [letter]. Ann Neurol 1997;42:12930.

19 Krack P, Pollak P, Limousin P, et al. Opposite motor effects of pallidal stimulation in Parkinson's disease. Ann Neurol 1998;43:180-92.

20 Pahwa R, Wilkinson S, Smith D, et al. High-frequency stimulation of the globus pallidus for the treatment of Parkinson's disease. Neurology 1997;49:249-53. 
21 Gross C, Rougier A, Guehl D, et al. High-frequency stimulation of the globus pallidus internalis in Parkinson's
disease: a study of seven cases. F Neurosurg 1997;87:491-8.

22 Wichmann T, DeLong M. Models of basal ganglia function and pathophysiology of movement disorders. Neurosurg Clin N Am 1998;9:223-36.

23 Tronnier VM, Krause M, Heck A, et al. Deep brain stimulation for the treatment of movement disorders. Neurol Psych Neurology, Psychiatry and Brain Research 1999;6:199-212.

24 Krack P, Benazzouz A, Pollak P, et al. Treatment of tremor in Parkinson's disease by subthalamic nucleus stimulation. Mov-Disord 1998;13:907-14.

25 Meyers H. Surgical procedure for postencephalic tremor. Archives of Neurology and Psychiatry 1940;44:455-7.

26 Narabayashi MD, Okuma T, Shikiba S. Procaine Oil Blocking of the Globus pallidus. Archives of Neurology and Psychiatry 1955;75:36-48.
27 Alberts WW, Feinstein B, Levin G, et al. Stereotaxic surgery for parkinsonism. Clinical results and stimulation threshfor parkinsonism. Clinical results
olds. $\mathcal{F}$ Neurosurg $1965 ; 23: 174-83$.

28 Levy A. Stereotaxic brain operations in Parkinson's syndrome and related motor disturbances. Comparison of lesions in the pallidum and thalamus with those in the internal capsule. Confinia Neurologica 1967;29(suppl):170

29 Fager CA. Evaluation of thalamic and subthalamic surgical lesions in the alleviation of Parkinson's disease. F Neurosurg 1968;28:145-9.

30 Velasco FC, Molina Negro P, Bertrand C, et al. Further definition of the subthalamic target for arrest of tremor. $\mathcal{F}$ Neurosurg 1972;36:184-91.

31 Vogel HP, Schiffter R. Hypersexuality: a complication of dopaminergic therapy in Parkinson's disease. Pharmacopsychiatria 1983;16:107-10. 